\title{
Paget disease of bone
}

\author{
G. David Roodman ${ }^{1,2}$ and Jolene J. Windle ${ }^{3}$
}

1Department of Medicine, Division of Hematology-Oncology, University of Pittsburgh Medical Center, Pittsburgh, Pennsylvania, USA. 2VA Pittsburgh Healthcare System, Medicine/Hematology-Oncology, Pittsburgh, Pennsylvania, USA. ${ }^{3}$ Department of Human Genetics, Virginia Commonwealth University, Richmond, Virginia, USA.

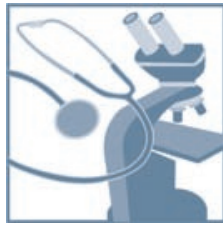

Paget disease of bone (PD) is characterized by excessive bone resorption in focal areas followed by abundant new bone formation, with eventual replacement of the normal bone marrow by vascular and fibrous tissue. The etiology of PD is not well understood, but one PD-linked gene and several other susceptibility loci have been identified, and paramyxoviral gene products have been detected in pagetic osteoclasts. In this review, the pathophysiology of PD and evidence for both a genetic and a viral etiology for PD will be discussed.

\section{Normal bone remodeling}

The normal adult skeleton undergoes constant remodeling, with osteoclasts removing bone and osteoblasts forming new bone at sites of previous bone resorption in a closely coupled fashion. Bone remodeling occurs in discrete areas, termed basic multicellular units, and it is estimated that the entire adult skeleton is remodeled every $2-4$ years $(1,2)$.

Osteoclasts are derived from mononuclear precursor cells in the monocyte-macrophage lineage, which fuse to form multinucleated osteoclasts that are then activated to resorb bone. Both systemic and local factors in the bone microenvironment play critical roles in the regulation of osteoclast formation and activity. In particular, receptor activator of NF-кB ligand (RANKL; also referred to as TRANCE, osteoclast differentiation-inducing factor, or osteoprotegerin [OPG] ligand), a member of the TNF superfamily, is a critical regulator of osteoclast formation (3-5). Most osteotropic factors, including $1,25-(\mathrm{OH})_{2} \mathrm{D}_{3}$, IL-1, IL-11, and parathyroid hormone (PTH), promote osteoclast formation indirectly by binding to marrow stromal cells and inducing expression of RANKL on their surface $(5,6)$. RANKL then binds the receptor activator of NF-KB (RANK) receptor on osteoclast precursors, leading to activation of a number of downstream signaling pathways, including the NF- $\mathrm{B}$, AKT, JNK, p38 MAPK, and ERK pathways. Each of these pathways has been implicated in osteoclast differentiation, function, or survival (6-9) (Figure 1). The importance of the RANKL-NF- $\mathrm{KB}$ signaling pathway in osteoclastogenesis has been highlighted by the finding that targeted disruption of multiple genes encoding components of this pathway (RANKL, RANK, TNF receptor-associated factor 6 [TRAF6], NF- $\mathrm{KB}$, and $\mathrm{NFATc}_{1}$, an NF-кB-activated transcription factor) in mice causes profound osteopetrosis $(5,9-17)$. However, additional RANK-activated signaling pathways are also clearly important regulators of osteoclasts, since both c-src- and c-fos-deficient mice display profound osteopetrosis, resulting from impaired osteoclast function in c-src-knockout mice (18) and from the failure of osteoclasts

Nonstandard abbreviations used: CBFA- 1 , core binding factor- $\alpha-1$; CDV, canine distemper virus; MV, measles virus; MVNP, MV nucleocapsid protein; OPG, osteoprotegerin; PD, Paget disease of bone; PTH, parathyroid hormone; RANK, receptor activator of NF-кB; RANKL, RANK ligand; SH2, Src homology 2; SSPE, subacute sclerosing panencephalitis; TRAF6, TNF receptor-associated factor 6; UBA,

ubiquitin-associating.

Conflict of interest: The authors have declared that no conflict of interest exists.

Citation for this article: J. Clin. Invest. 115:200-208 (2005)

doi:10.1172/JCI200524281. to form in the c-fos knockouts (19). In addition, the transcription factor PU.1, which is involved in the process of commitment of hematopoietic stem cells, is critical for normal osteoclast formation, since fetal mice lacking this gene do not form osteoclasts (20).

TNF and IL-1 activate many of the same downstream signaling pathways as does RANKL (Figure 1), and both of these cytokines have been shown to play a role in the regulation of osteoclast differentiation and function (21-27). However, neither cytokine appears to be as central to the process of osteoclastogenesis as RANKL, since disruption of either the TNF or IL-1 receptors in mice results in a minimal bone phenotype, as compared with that of the RANKor RANKL-knockout mice (28-32). Further, to date, neither IL-1 nor TNF has been implicated in the pathogenesis of PD.

Once bone resorption within a basic multicellular unit is complete, osteoblast precursors are then recruited to the site of previous bone resorption and differentiate to become bone-forming cells. Osteoblasts are derived from mesenchymal stem cells, which can form osteoblasts, osteocytes, or muscle cells (1). The transcription factor RUNX-2, also known as core binding factor- $\alpha-1$ (CBFA-1), is critical for the differentiation of osteoblasts, since bone does not develop in mice lacking CBFA-1 $(33,34)$. Osteoblasts ultimately terminally differentiate into osteocytes, which are trapped in the calcified bone matrix. Regulators of osteoblast differentiation include the bone morphogenetic proteins, insulin-like growth factors, TGF- $\beta$, fibroblast growth factors, and platelet-derived growth factors (35-38).

\section{Abnormal bone remodeling in Paget disease}

Paget disease of bone (PD) is the second most common bone disease after osteoporosis (39). The disease is characterized by focal regions of highly exaggerated bone remodeling, with abnormalities in all phases of the remodeling process. The majority of patients with PD are elderly, with the age at diagnosis usually more than 50 years. It affects both males and females, with a slight predominance in males. Although PD is often asymptomatic, $10-30 \%$ of patients experience pain, skeletal deformity, neurologic symptoms, pathologic fractures, or deafness (39). Table 1 lists the common symptoms and findings in Paget patients. Patients may have only one affected bone or have pagetic lesions in multiple bones. However, PD remains highly localized, and patients rarely develop new lesions in previously unaffected bones after diagnosis (40). The most serious complication of PD is development of osteosarcoma in the pagetic bone, although this is relatively rare, occurring in less than $1 \%$ of patients $(41,42)$. 


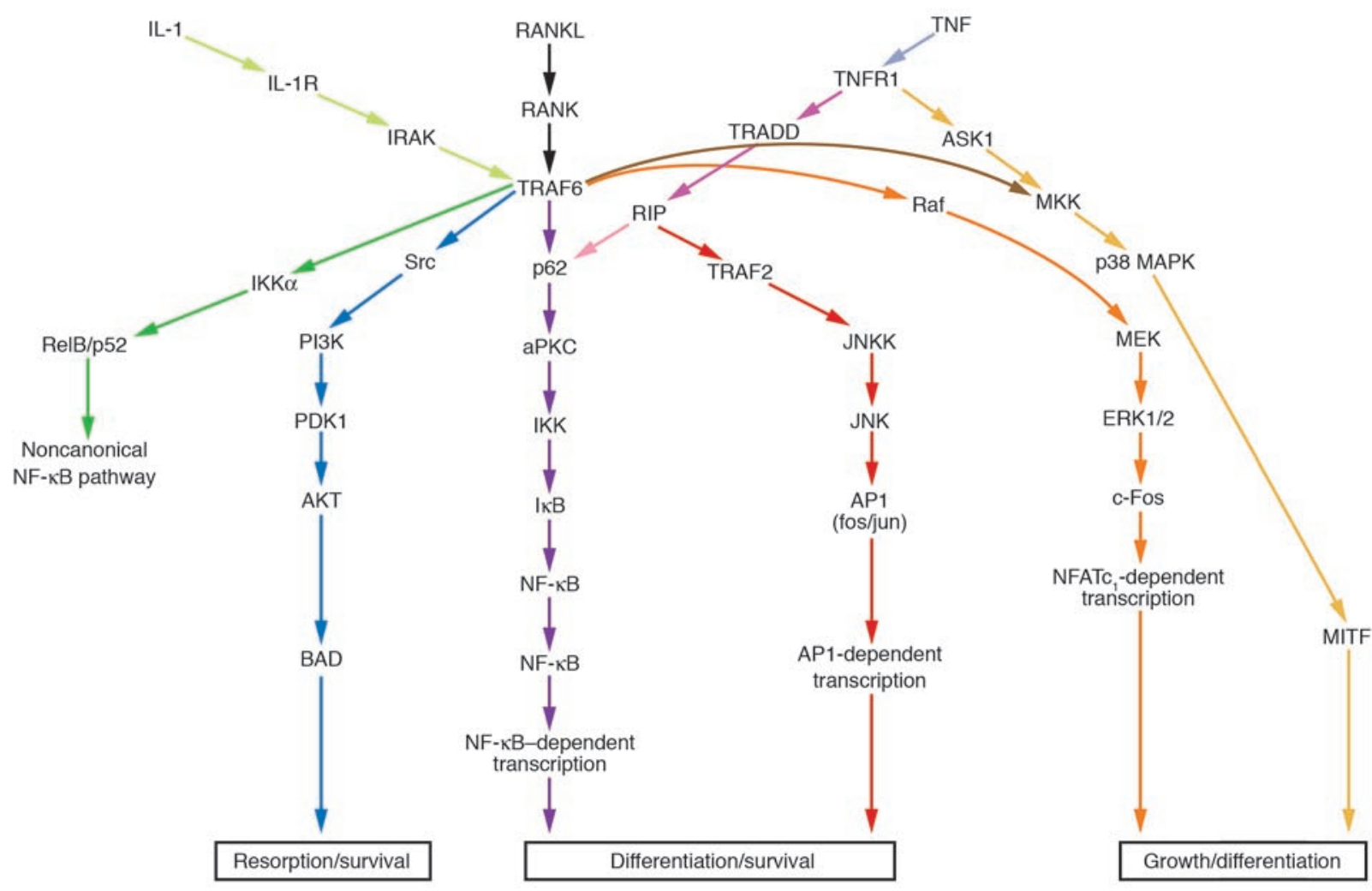

Figure 1

Signaling pathways involved in osteoclast formation and activity. When RANKL binds RANK, multiple signaling pathways can be activated, including NF-KB, AKT, JNK, p38 MAPK, and ERK, resulting in subsequent activation of genes that regulate osteoclast formation, bone resorption, and survival. TRAF6 appears to play a central role in the activation of most of these pathways. AP1, activator protein 1; aPKC, atypical PKC; IKB, inhibitor of $\kappa \mathrm{B}$; ASK1, apoptosis signal-regulating kinase 1; BAD, Bcl-2-associated death promoter; IL-1R, IL-1 receptor; IKK $\alpha$, IKB kinase $\alpha$; IRAK, IL-1 receptor-associated kinase; JNKK, JNK kinase; MEK, MAPK/ERK kinase; MITF, microphthalmia transcription factor; MKK, MAPK kinase; NFATc $c_{1}$, nuclear factor of activated T cells cytoplasmic 1; PDK1, phosphoinositide-dependent protein kinase 1; RIP, receptor interacting protein; TNFR1, TNF receptor 1; TRADD, TNF receptor 1-associated death domain.

The initial phase of PD is characterized by excessive bone resorption in a focal region, and radiological examination in the early stages of the disease frequently shows an osteolytic lesion. Subsequently, bone formation is also markedly increased, with increased numbers of osteoblasts that appear hyperactive but normal morphologically. The increased population of osteoblasts rapidly deposits new bone in a chaotic fashion so that the bone formed in pagetic lesions is of poor quality and is disorganized rather than lamellar in character (Figure 2). The poor quality of pagetic bone accounts for the bowing or even fracture of bones affected by PD. As rapid bone formation predominates in the more advanced stages of $\mathrm{PD}$, the lesions become sclerotic, with observed replacement of the bone marrow with vascular and fibrous tissue and thickening of the bone (43).

Blood chemistries of Paget patients usually reflect the increased bone remodeling, with elevated levels of both bone resorption and formation markers (see Biochemical markers of bone remodeling that are increased in $P D$ ). For example, the level of alkaline phosphatase in the serum, which reflects osteoblast activity, and N-telopeptide of type I collagen in the urine, which is released during bone resorption and reflects osteoclast activity, can both be markedly elevated (up to 10- to 20-fold) in patients with PD (44). Because bone resorption and formation remain coupled in PD, there is a high correlation between the levels of bone resorption and bone formation markers in Paget patients.

\section{Osteoclasts in PD}

The osteoclast is the primary cell affected by PD. Osteoclasts in pagetic lesions are increased in both number and size (43), and in cross-section are seen to contain up to 100 nuclei, in contrast to normal osteoclasts, which contain 3-20 nuclei (Figure 3, A and B). A striking feature of pagetic osteoclasts is the characteristic nuclear inclusions, which consist of paracrystalline arrays that are similar to nucleocapsids of paramyxoviruses (Figure 3C) (45). These nuclear inclusions are not present in other bone marrow cells in the pagetic lesion or in nonpagetic bone in patients with PD. Similar nuclear inclusions have been reported in osteoclasts from patients with oxalosis, osteopetrosis, and giant cell tumors of bone (46-48), but this is not a consistent finding in these conditions, as it is in PD.

In addition to the morphologic abnormalities in pagetic osteoclasts, osteoclast precursors are physiologically abnormal. In vitro studies of bone marrow samples obtained from affected bones of Paget patients have identified several unique differences between pagetic and normal osteoclast precursors. This "pagetic phenotype" is characterized by hypersensitivity of osteoclast precursors to several osteoclastogenic factors, including $1,25-(\mathrm{OH})_{2} \mathrm{D}_{3}$ $(49,50)$ and RANKL $(50,51)$. Osteoclasts precursors in bone marrow cultures obtained from pagetic lesions form osteoclasts at concentrations of these factors that are 10- to 100-fold lower than levels required for normal osteoclast formation. In addition, the level 


\section{Table 1}

Clinical presentation in PD

\begin{tabular}{|c|c|}
\hline Symptom & Etiology \\
\hline Bone pain & $\begin{array}{l}\text { Usually results from osteoarthritis in joints } \\
\text { adjacent to pagetic bones }\end{array}$ \\
\hline Bone deformities & $\begin{array}{l}\text { Can result in bowing of a limb or increased skull } \\
\text { size due to rapid formation of poor-quality bone }\end{array}$ \\
\hline Fracture & $\begin{array}{l}\text { Bone in pagetic lesions is weaker than normal } \\
\text { bone and can develop characteristic "chalk } \\
\text { stick-like" fractures }\end{array}$ \\
\hline Hearing loss & Temporal bone involvement \\
\hline $\begin{array}{l}\text { Nerve root } \\
\text { compression }\end{array}$ & $\begin{array}{l}\text { Impingement of nerve root by increased bone } \\
\text { formation }\end{array}$ \\
\hline Headache & Skull affected by PD \\
\hline
\end{tabular}

of $\mathrm{TAF}_{\mathrm{II}}-17$, a component of the $\mathrm{TAF}_{\mathrm{II}} \mathrm{D}$ transcription factor complex that binds the vitamin $\mathrm{D}$ receptor, is increased in osteoclast precursors from affected bones of Paget patients as compared with normal osteoclast precursors (52). The increased level of $\mathrm{TAF}_{\mathrm{II}}-17$ appears to be responsible, in part, for the hypersensitivity of pagetic osteoclast precursors to $1,25-(\mathrm{OH})_{2} \mathrm{D}_{3}(52)$.

\section{Treatment of PD}

Since the osteoclast is the primary cell affected by PD, treatment has been directed at inhibiting osteoclast formation or osteoclastic bone resorption or inducing osteoclast apoptosis. Table 2 lists agents that are currently used for the treatment of PD. Calcitonin was initially used to treat patients with PD because it inhibits osteoclastic bone resorption and osteoclast formation (53). Calcitonin can induce remission in patients with $\mathrm{PD}$, but more than $50 \%$ of patients treated with salmon calcitonin for more than 6 months develop calcitonin antibodies, and 10-20\% become resistant to calcitonin. Bisphosphonates, which block osteoclast formation and induce osteoclast apoptosis (54), have supplanted calcitonin as the treatment of choice for PD. First-generation bisphosphonates, such as etidronate, can induce partial or complete remissions in PD patients, and with the development of more potent bisphosphonates, patients can experience prolonged remissions, lasting months to years. In patients with more extensive PD involving many bones, intravenous bisphosphonates such as pamidronate or zoledronate may be used. However, neither calcitonin nor bisphosphonates cure PD; they only control the disease process. Treatment of PD is indicated to control bone pain, prevent fractures, minimize bleeding prior to surgery on a pagetic bone, and decrease local progression in weight-bearing bones or the skull.

\section{Etiology of PD}

Genetics of PD. The cause of PD is currently an area of intensive investigation, and both genetic and nongenetic factors have been implicated in the pathogenesis of this disease. Genetic factors are clearly an important component of the etiology of PD, since $15-40 \%$ of affected patients have a first-degree relative with PD (55), and numerous studies have described extended families with PD exhibiting an autosomal dominant mode of inheritance (56-58). Although familial PD was initially thought to have a very high penetrance, recent studies have suggested that the penetrance is highly variable (59). Ethnic differences in the incidence of PD have been noted, and these persist with emigration to other locales. For example, PD is common in persons of Anglo-Saxon origin, but the prevalence is low in the Far East and does not change when populations from this region move to areas of higher prevalence, such as the United Kingdom (60).

Several susceptibility loci for PD have been recently identified, including 2q36, 5q31, 5q35, 10p13, 18q21-22, and 18q23 (61-66) (Table 3). Mutations in the TNFRSF11A gene (encoding RANK) on chromosome 18q21-22 have been linked to familial expansile osteolysis, a rare bone disease that shares many clinical features with but is distinct from PD (67). In addition, a TNFRSF11A mutation was identified in an Asian family with early-onset PD (68). However, RANK mutations have not been observed in patients with the more typical form of $\mathrm{PD}$, which occurs predominately in elderly patents and rarely occurs in Asians. In 2002 Laurin et al. reported a point mutation (P392L) in SQSTM1, which maps to chromosome 5q35, in two French Canadian Paget families and several unrelated patients (69). SQSTM1 encodes sequestosome 1, also known as $\mathrm{p} 62$, which is a ubiquitin-binding protein that is involved in the IL-1, TNF, and RANKL signaling pathways (Figure 1). Subsequently, other groups have identified additional mutations in p62 in both familial and nonfamilial PD, including both amino acid substitutions and mutations that result in total deletion of the ubiquitin-binding domain (70-72). SQSTM1 is the gene most frequently linked to $\mathrm{PD}$, and mutations of this gene have been detected in up to $30 \%$ of familial Paget cases studied.

p62 was first identified as a protein that binds to the Src homology 2 (SH2) domain of $\mathrm{p} 56^{\text {lck }}$ in a phosphotyrosine-independent manner (73). It was named sequestosome 1 because it forms a cytoplasmic complex with ubiquitinated proteins $(74,75)$. The SQSTM1 gene is highly conserved, especially in the $\mathrm{COOH}$-terminal region of the protein. This region of the protein interacts noncovalently with polyubiquitin chains and shares structural homology with other proteins containing ubiquitin-associating (UBA) domains. Interestingly, all the PD-associated mutations in p62 identified to date are located in this region (Figure 4). Patients with truncation mutations in $\mathrm{p} 62$ exhibit a more severe Paget phenotype than patients with any of the point mutations $(72,76)$. However, it is not yet clear whether alterations in ubiquitin binding are directly related to the
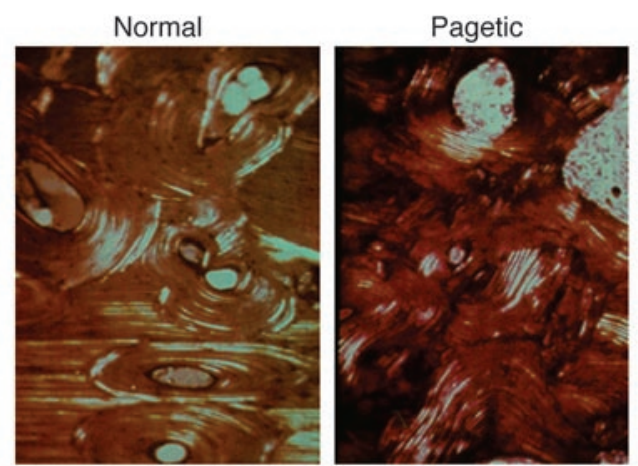

\section{Figure 2}

Normal and pagetic bone. Normal (A) and pagetic (B) bone are shown under polarized light. Pagetic bone is poorly organized and very chaotic in structure and forms "a mosaic pattern." In contrast, normal bone is highly organized with a lamellar structure. Figure reproduced with permission from The Paget's Foundation for Paget's Disease of Bone Related Disorders. 


\author{
Biochemical markers of bone remodeling \\ that are increased in PD \\ Markers of bone resorption \\ Urinary hydroxyproline \\ Serum N-telopeptide of type I collagen \\ Serum C-telopeptide of type I collagen \\ Serum deoxypyridinoline cross-links of type I collagen \\ Markers of bone formation \\ Serum alkaline phosphatase \\ Serum bone-specific alkaline phosphatase \\ Osteocalcin \\ Serum N-terminal propeptide of type I collagen
}

mechanism of pathogenesis. In addition to the UBA domain, the p62 protein is characterized by several other motifs that mediate protein-protein interactions that are relevant to signaling pathways involved in osteoclastogenesis, including an atypical PKC-interacting domain, a $\mathrm{Zn}$-finger domain that mediates binding to regulated intramembrane proteolysis, and a TRAF6-interacting domain (77).

As shown in Figure 1, p62 plays a critical role in multiple signaling pathways that regulate osteoclastogenesis. Duran and coworkers reported that the $\mathrm{P} 392 \mathrm{~L}$ mutation in $\mathrm{p} 62$ that is linked to $\mathrm{PD}$ results in enhanced NF- $\mathrm{\kappa B}$ signaling (78), although the mechanism responsible for this remains to be determined. The PD-associated p62 mutations could potentially affect a number of cell processes, including signaling, ubiquitin-dependent proteolysis, and others.

We have recently shown in preliminary studies that transfection of normal human osteoclast precursors with a P392L mutant p62 construct enhanced the sensitivity of normal human osteoclast precursors to RANKL and increased osteoclast formation (79). Interestingly, we observed neither hypersensitivity of the precursors to $1,25-(\mathrm{OH})_{2} \mathrm{D}_{3}$ nor an increased number of nuclei per osteoclast in the osteoclasts that formed in vivo, both of which are characteristics of pagetic osteoclasts. We have also targeted the P392L mutant p62 gene to cells in the osteoclast lineage of transgenic mice using the tartrate resistant acid phosphatase (TRAP) promoter, which directs expression to both osteoclasts and osteoclast precursors. Initial characterization of these mice showed that they have increased osteoclast numbers and are osteopenic but do not develop the increased osteoblast activity that is characteristic of pagetic lesions. These preliminary in vitro and in vivo studies suggest that the P392L mutation in p62 enhances osteoclast formation, possibly through increased RANK signaling. The increased osteoclast activity caused by mutations in the p62 gene may explain the increased bone turnover that has been observed in some Paget patients in bones not affected by PD (80).

Studies of families with PD linked to mutations in the p62 gene also suggest that these mutations may not completely account for the pathogenesis of PD. The severity of disease in family members carrying the same mutation can vary widely, and up to $20 \%$ of individuals who harbor p62 mutations and are older than

\section{Figure 3}

55 years do not have PD (81). These data suggest that additional factors may be affected by the pathogenesis of PD.

A number of additional candidate genes have been evaluated to determine whether they might be linked to $\mathrm{PD}$, including genes known to be involved in osteoclast biology or genes whose deletion results in mice that display an osteoclast phenotype. For example, the gene encoding OPG, a decoy receptor for RANKL, is on chromosome 18q24.2. OPG decreases osteoclast formation by binding to RANKL and interfering with its binding to the RANK receptor (3). Mutations in OPG have been reported in patients with idiopathic hyperphosphatasia, a rare congenital disorder that occurs in childhood and is characterized by deafness and bone lesions that affect the entire skeleton (82), but OPG mutations have not been reported in adults with PD. However, it is likely that additional genes linked to PD will be identified, and it is reasonable to hypothesize that they may be involved in the regulation of osteoclast formation, function, or lifespan, since increased osteoclast activity is central to PD. These genes may be components of the RANK or other signaling pathways that control osteoclast formation or may result in increased expression of transcription factors such as c-fos or NFATc $c_{1}$ that are critical for osteoclastogenesis.

\section{Potential viral etiology of PD}

Several observations suggest that environmental factors may also contribute to the pathogenesis of $\mathrm{PD}$. The variable penetrance of $\mathrm{PD}$ within families with a genetic predisposition to $\mathrm{PD}$, the observation that the disease remains highly localized to a particular bone or bones rather than affecting the entire skeleton, and the fact that the incidence and severity of the disease has been changing over the last 25 years $(83,84)$ all support the hypothesis that additional, nongenetic factors are involved in the development of PD. PD affected approximately $2-8 \%$ of the population in the United Kingdom and New Zealand 20 years ago, but recent studies of the prevalence of PD in subjects of European origin in $2 \mathrm{New}$ Zealand cities found that the prevalence of PD was about half of what had been estimated 25 years ago (84). Similarly, Van Staa and coworkers recently conducted a radiologic survey in 10 British cities and found a decrease in the incidence of PD compared with the findings of the original studies performed some 20 years earlier (83). These reports suggest that an additional, nongenetic factor(s)
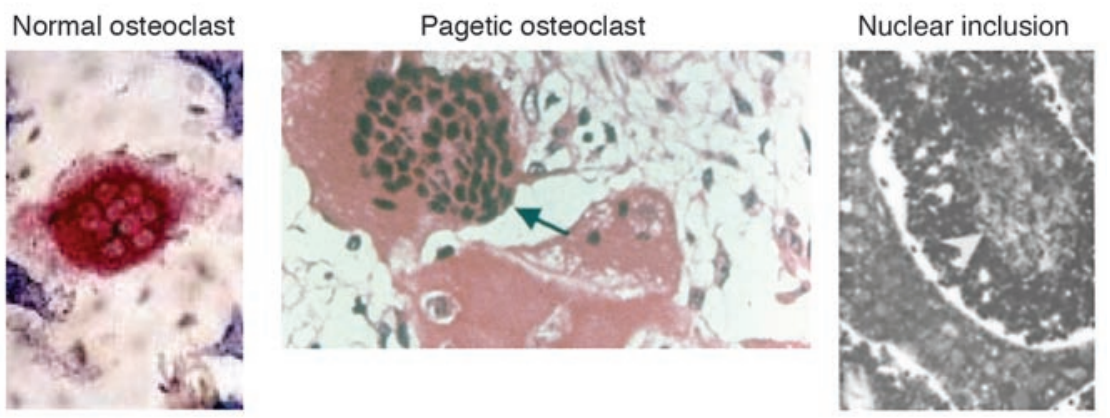

Osteoclasts in normal bone and in Paget's disease. (A) Normal osteoclasts are large multinucleated cells that contain between 3 and 20 nuclei per cell. (B) In contrast, pagetic osteoclasts are markedly increased in number and size and can contain up to 100 nuclei (arrow). (C) On ultrastructural examination, pagetic osteoclasts have characteristic nuclear (arrowhead) and occasional cytoplasmic inclusions containing paracrystalline arrays that are similar to paramyxoviral nucleocapsids. 


\section{Table 2}

PD therapies

$\begin{array}{ll}\text { Agent } & \text { Dosage } \\ \text { Calcitonin } & 50-100 \text { units subcutaneously daily for 6-18 months } \\ \text { Etidronate } & 20-400 \mathrm{mg} \text { orally daily for } 6 \text { months } \\ \text { Pamidronate } & 30-90 \mathrm{mg} \text { intravenously daily for } 1-2 \text { days } \\ \text { Alendronate } & 40 \mathrm{mg} \text { orally daily for } 6 \text { months } \\ \text { Tiludronate } & 400 \mathrm{mg} \text { orally daily for } 3 \text { months } \\ \text { Risedronate } & 30 \mathrm{mg} \text { orally daily for } 2 \text { months }\end{array}$

may be involved in the initiation of the disease process in patients with a genetic predisposition to PD.

Ultrastructural, immunohistochemical, in situ hybridization, and biological studies have all suggested a possible viral etiologic factor in PD, although an infectious virus has not been isolated. Abe et al. reported that budding viruses could be detected in osteoclasts from PD patients (85), but this has not been confirmed by other investigators. Mills and coworkers demonstrated that the nuclear inclusions observed in osteoclasts from PD patients cross-reacted with antibodies against respiratory syncytial virus and measles virus nucleocapsid protein (MVNP) (86). Basle and coworkers reported that MVNP mRNA was present in osteoclasts from 5 patients with PD but not in 3 control patients (87). Similarly, Mills and coworkers (86) found that MVNP protein was present in osteoclasts and/or cultured bone cells from patients with PD. In bone biopsy specimens, both MVNP and respiratory syncytial virus nucleocapsid proteins were detected in the same osteoclasts on serial sections. Basle and coworkers (88) also detected MVNP in 6 of 6 specimens isolated from patients with PD but found other paramyxoviral nucleocapsid proteins as well. Mills and coworkers (89) studied long-term marrow cultures of samples from 12 patients with PD and found that in all 12 cultures, MVNP and/or syncytial virus nucleocapsid proteins were present in the mononuclear cells or the osteoclast-like multinucleated cells that formed. In contrast, these viral proteins were detected in less than $5 \%$ of osteoclast-like cells from control subjects.

Reddy et al. detected MVNP transcripts in bone marrow samples obtained from affected bones from 9 of 10 patients with PD (90). More recently, Friedrichs et al. reported the full-length sequence of an MVNP gene isolated from marrow cells of a Paget patient, as well as 700 base pairs of MVNP sequence from 3 other patients (91). Together, these data support the hypothesis that the MVNP gene is present in osteoclasts from patients with PD. However, this is not a universal finding, since others have been unable to detect the presence of paramyxoviral transcripts in either freshly isolated bone marrow specimens, osteoclasts, or cultured marrow cells from Paget patients $(92,93)$. Further, none of these studies have demonstrated that a virus is the cause of PD. Importantly, prior to the era of measles virus (MV) immunization, measles was a ubiquitous infection, while PD has a distinct geographic and racial distribution. PD is rare in the Far East and Scandinavia but is relatively common in the United Kingdom, Australia, New Zealand, and the United States. These results suggest that if involved, a viral infection by itself does not cause PD.

Our laboratory has undertaken a series of studies to determine whether MV could induce pagetic-like osteoclasts and bone lesions. MV consists of 6 genes: the nucleocapsid, matrix, fusion, hemagglutinin, and the $\mathrm{P}$ and $\mathrm{L}$ genes, which constitute the viral polymerase. Kurihara et al. showed that transfection of normal human osteoclast precursors with the MVNP gene, but not the matrix or fusion gene, resulted in the formation of osteoclasts that exhibited many of the characteristics of pagetic osteoclasts (94). These characteristics included increased rate of osteoclast formation, increased numbers and size of osteoclasts formed in vitro, increased bone resorbing capacity of the osteoclasts, hypersensitivity of transfected osteoclast precursors to $1,25-(\mathrm{OH})_{2} \mathrm{D}_{3}$, and increased expression levels of $\mathrm{TAF}_{\mathrm{II}}-17$. These characteristics are also observed in osteoclasts formed in vitro from freshly isolated marrow samples from Paget patients (94). Further, MV infection of marrow cells from transgenic mice in which expression of the human MV receptor CD46 was targeted to cells in the osteoclast lineage resulted in formation of osteoclasts that had many of the characteristics of Paget osteoclasts (95) (normal mice do not express MV receptors and are resistant to MV infection).

Additional support for a potential role for MVNP in the pathogenesis of PD is provided by our preliminary studies, in which expression of the MVNP gene was targeted to the cells in the osteoclast lineage in transgenic mice (MVNP mice) (96). Histomorphometric analysis of bones from $17 \mathrm{MVNP}$ and 16 wild-type mice examined between 3 and 14 months of age showed there was a significant increase in osteoclast numbers and osteoblast activity in MVNP mice. This was accompanied by a marked increase in the amount of woven bone and in the cancellous bone volume. In contrast, bone volume decreased between 3 and 14 months of age in wild-type mice. Ex vivo studies showed that the osteoclasts formed in marrow cultures from MVNP mice were increased in number, were hypersensitive to $1,25-(\mathrm{OH})_{2} \mathrm{D}_{3}$, and had an increased bone resorbing capacity compared with wild-type osteoclasts in culture. These results suggest that expression of MVNP in osteoclasts in vivo can induce bone changes that share many of the features of PD.

However, several questions remain concerning the involvement of $\mathrm{MV}$ in the pathogenesis of $\mathrm{PD} . \mathrm{MV}$ infections predominantly occur in children rather than in adults, while PD is usually diagnosed in elderly patients. The osteoclast is not a self-renewing cell but is formed by fusion of postmitotic precursors. Thus, cell types other than osteoclasts must serve as a reservoir for MV to persist for long periods of time in patients with PD. Reddy et al. have reported that cells of other hematopoietic lineages from Paget disease patients, including immature multipotent precursors that give rise to granulocytes, erythrocytes, macrophages, and platelets, also express MVNP transcripts (97). These results suggest that the pluripotent hematopoietic stem cell may be the initial target for MV infection in PD.

Persistent paramyxoviral infections do occur. Chronic MV infection of the nervous system has been reported in patients with sub-

\section{Table 3}

Genetic loci linked to PD

\begin{tabular}{lcc}
\hline & & \\
Locus & Gene & Protein affected \\
$2 \mathrm{q} 36$ & $?$ & $?$ \\
$5 \mathrm{q} 31$ & $?$ & $?$ \\
$5 \mathrm{q} 35$ & SQSTM1 & $\mathrm{p} 62$ \\
$6 \mathrm{p}$ & $?$ & $?$ \\
$10 \mathrm{p} 13$ & $?$ & $?$ \\
$18 \mathrm{q} 21-22$ & TNFRSF11A & RANK \\
$18 \mathrm{q} 23$ & $?$ & $?$ \\
\hline
\end{tabular}




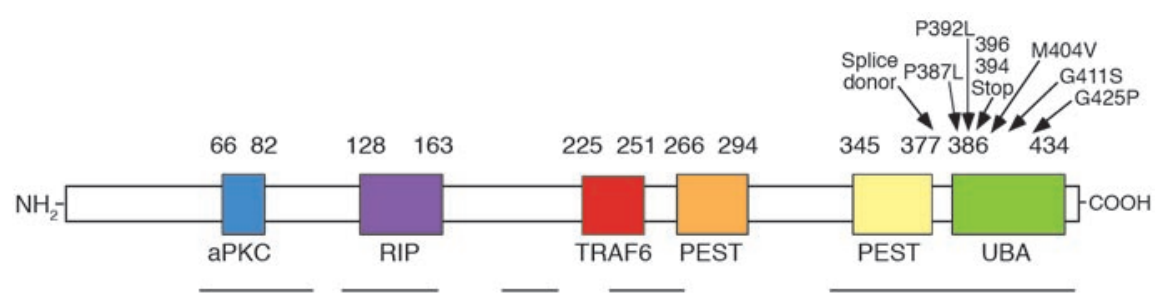

Figure 4

Structure of the p62 protein. The blocks indicate domains that mediate association with other proteins or are hypothesized to mediate these associations based upon homology with other proteins. The solid lines below the protein indicate stretches of sequence identity (of 20 amino acids or more) among the mouse, rat, and human p62 proteins. The arrows above the protein indicate the Paget disease-associated mutations identified to date. The splice donor and stop mutations result in a truncated protein lacking the UBA domain. PEST denotes hydrophobic regions that target proteins for rapid degradation (P, proline; E, glutaric acid; S, serine; $T$, threonine).

acute sclerosing panencephalitis (SSPE), which develops usually 5 years after the onset of a classic MV infection (98). However, as with PD patients, it has been difficult to rescue infectious virus from SSPE patients. Other RNA viruses can also persist in vivo, including influenza virus (99) and swine vesicular disease virus (100), and result in a carrier state in which the virus cannot be detected or is asymptomatic for long periods of time.

Several groups have also investigated the possible association of another paramyxovirus, canine distemper virus (CDV), with PD. An epidemiologic study in England suggested that patients with PD were more likely to have a pet dog than non-PD controls (101). Gordon and colleagues found that bone specimens from 11 of 25 Paget patients in England expressed CDV mRNA according to in situ hybridization analysis (102), and they also amplified an RT-PCR product for the CDV nucleocapsid gene from pagetic bone cells. Using in situ PCR techniques, Mee and coworkers also detected CDV nucleocapsid transcripts in osteoclasts from bone biopsies from 12 of 12 Paget patients in England (103). Taken together, these studies demonstrate that paramyxoviruses can induce changes in osteoclasts and bone that are similar to those found in PD. However, the role of paramyxoviral transcripts or proteins in the etiology of PD remains controversial.

\section{Other factors that may be involved in the development of PD}

Several studies have reported increased levels of IL-6 and/or $\mathrm{M}-\mathrm{CSF}$ in patients with PD (104-106). Osteoclasts formed in bone marrow cultures from patients with PD secrete large quantities of IL- 6 into the conditioned media, with IL- 6 levels reaching up to $2,000 \mathrm{pg} / \mathrm{ml}$ (104). IL-6 levels are also increased in the bone marrow plasma of affected bones from Paget patients, as well as in their peripheral blood (104). Since IL-6 has been shown to induce osteoclast formation (107), it is possible that IL-6 plays a role in the enhanced osteoclast formation in PD. Alternatively, the increased levels of IL-6 seen in patients with PD may simply be a marker for the increased osteoclast formation.

Athanasou and coworkers reported that serum levels of M-CSF are also increased in Paget disease patients at diagnosis and fall when the patients are treated effectively with bisphosphonates (106). M-CSF in combination with RANKL is a critical factor for osteoclast formation (108), and rodents deficient in M-CSF develop osteopetrosis $(108,109)$. The increased levels of M-CSF in PD may reflect the increased numbers of osteoblasts present in the pagetic lesion, since osteoblasts produce M-CSF (110). When PD patients are in remission, osteoblast activity decreases, and M-CSF levels would be expected to fall accordingly. It is possible that the increased levels of IL-6 and M-CSF together could further increase osteoclast activity in the pagetic lesion, thereby amplifying the pagetic process.

\section{A proposed model for the development of PD}

Any model for the development of PD must take into account both genetic and nongenetic factors, the highly localized nature of the disease, and its late onset. The involvement of a nongenetic factor in the etiology of PD would explain why some individuals

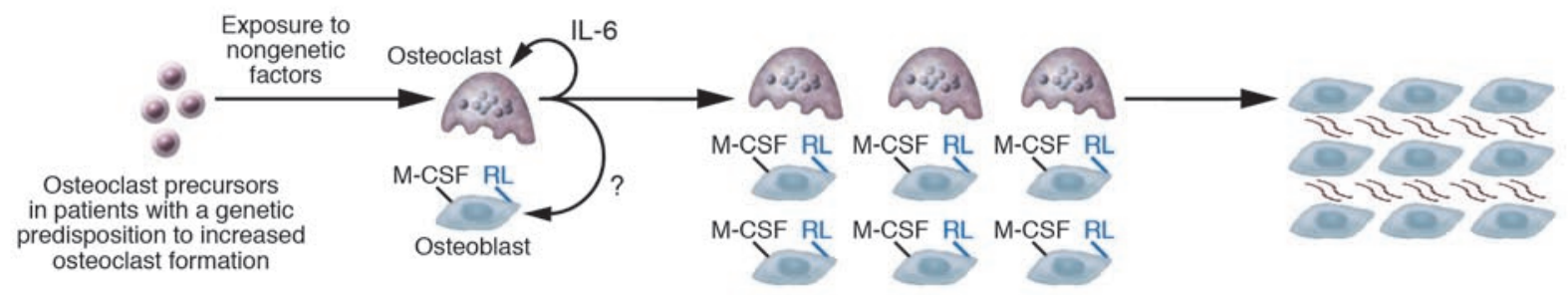

Figure 5

A proposed model for the pathogenesis of PD. Mutations that enhance basal osteoclastogenesis predispose patients to PD by creating a permissive environment for its development. A second factor, such as expression of certain viral proteins, may further alter signaling pathways or expression of specific transcription factors, resulting in the abnormal characteristics of pagetic osteoclasts. For example, the increased sensitivity of osteoclast precursors to low levels of $1,25-(\mathrm{OH})_{2} \mathrm{D}_{3}$ and RANKL (RL) enhances osteoclast formation. Further, the increased numbers of osteoclasts would secrete high levels of IL-6, which would further enhance osteoclast formation. Since osteoclast and osteoblast activity remain coupled in PD, the increased osteoclast activity would result in increased osteoblast numbers and rapid formation of new bone. The increased numbers of immature osteoblasts expressing high levels of RANKL and M-CSF would further increase osteoclast formation. As more and more bone is formed, the lesion would eventually become sclerotic. 
who have a PD-associated mutation, such as a P392L mutation in the SQSTM1 gene, do not develop PD. One possibility is that such mutations predispose patients to $\mathrm{PD}$, perhaps by enhancing basal osteoclastogenesis, thereby creating a permissive environment for the development of PD. A second factor, such as expression of certain viral proteins, may further alter signaling pathways or expression of specific transcription factors, resulting in the abnormal characteristics of pagetic osteoclasts. These include changes in the vitamin $\mathrm{D}$ receptor transcription complex and changes in NF- $\mathrm{KB}$ signaling and other signaling pathways that increase osteoclast formation. For example, the increased sensitivity of osteoclast precursors to low levels of $1,25-(\mathrm{OH})_{2} \mathrm{D}_{3}$ and RANKL would enhance osteoclast formation. Further, the increased numbers of osteoclasts would secrete high levels of IL-6, which would further enhance osteoclast formation. Since osteoclast and osteoblast activity remain coupled in $\mathrm{PD}$, the increased osteoclast activity would result in increased osteoblast numbers and rapid formation of new bone. The increased numbers of immature osteoblasts expressing high levels of RANKL and M-CSF would further increase osteoclast formation. As more and more bone is formed, the lesion would eventually become sclerotic. This model for the pathogenesis of PD is depicted in Figure 5.

\section{Summary}

There has been a tremendous output of new information on the pathogenesis of PD in recent years. Identification of genes involved in osteoclastogenesis that are mutated in PD and the characterization of other nongenetic factors that may be involved have provided important insights into the control of bone remodeling in $\mathrm{PD}$ as well as in normal bone. Future studies of the abnormal bone remodeling in PD may result in the identification of "coupling factors," which link osteoclastic bone resorption to new bone formation. The development of animal models of PD should greatly aid in these studies. If these coupling factors are identified, they may be useful in developing treatments for other diseases associated with bone destruction, such as bone metastasis and osteoporosis. New therapies under development for inhibiting osteoclast formation and bone resorption, such as antibodies to RANKL and inhibitors of cathepsin $\mathrm{K}$, the enzyme secreted by osteoclasts that degrades bone matrix and is required for bone resorption (111), may be useful treatments for PD. Vitamin D receptor antagonists, which could decrease the hypersensitivity of pagetic osteoclast precursors in PD patients to physiologic levels of vitamin D, may also be therapeutic avenues to pursue. Thus, understanding of the pathophysiology of PD should provide important insights into the mechanisms that control normal osteoclast differentiation and osteoblast formation and may lead to development of new anabolic therapies for treating patients with severe bone loss.

\section{Acknowledgments}

Studies by the author's laboratory are supported by research funds from the NIH (grant PO1-AR049363). We also thank Donna Gaspich for preparation of the manuscript and Frederick Singer (John Wayne Cancer Institute, Santa Monica, California, USA) for helpful discussions.

Address correspondence to: G. David Roodman, VA Pittsburgh Healthcare System, Medicine/Hematology-Oncology (111-H), University Drive, Pittsburgh, Pennsylvania 15240, USA. Phone: (412) 688-6571; Fax: (412) 688-6960; E-mail: roodmangd@upmc.edu.
1. Jilka, R.L. 2003. Biology of the basic multicellular unit and the pathophysiology of osteoporosis. Med. Pediatr. Oncol. 41:182-185.

2. Parfitt, A.M. 2002. Targeted and nontargeted bone remodeling: relationship to basic multicellular unit origination and progression. Bone. 30:5-7.

3. Yasuda, H., et al. 1998. Osteoclast differentiation factor is a ligand for osteoprotegerin / osteoclastogenesis-inhibitory factor and is identical to TRANCE/RANKL. Proc. Natl. Acad. Sci. U. S. A. 95:3597-3602.

4. Lacey, D.L., et al. 1998. Osteoprotegerin ligand is a cytokine that regulates osteoclast differentiation and activation. Cell. 93:165-176.

5. Kong, Y.Y., et al. 1999. OPGL is a key regulator of osteoclastogenesis, lymphocyte development and lymph-node organogenesis. Nature. 397:315-323.

6. Horwood, N.J., Elliott, J., Martin, T.J., and Gillespie, M.T. 1998. Osteotropic agents regulate the expression of osteoclast differentiation factor and osteoprotegerin in osteoblastic stromal cells. Endocrinology. 139:4743-4746.

7. Hofbauer, L.C., and Heufelder, A.E. 2001. Role of receptor activator of nuclear factor-kappaB ligand and osteoprotegerin in bone cell biology. J. Mol. Med. 79:243-253.

8. Lee, S.E., et al. 2002. The phosphatidylinositol 3-kinase, p38, and extracellular signal-regulated kinase pathways are involved in osteoclast differentiation. Bone. 30:71-77.

9. Kim, N., Odgren, P.R., Kim, D.K., Marks, S.C.J., and Choi, Y. 2002. Diverse roles of the tumor necrosis factor family member TRANCE in skeletal physiology revealed by TRANCE deficiency and partial rescue by a lymphocyte-expressed TRANCE transgene. Proc. Natl. Acad. Sci. U. S. A. 97:10905-10910.

10. Dougall, W.C., et al. 1999. RANK is essential for osteoclast and lymph node development. Genes
Dev. 13:2412-2424.

11. Li, J., et al. 2000. RANK is the intrinsic hematopoietic cell surface receptor that controls osteoclastogenesis and regulation of bone mass and calcium metabolism. Proc. Natl. Acad. Sci. U. S. A. 97:1566-1571.

12. Naito, A., et al. 1999. Severe osteopetrosis, defective interleukin-1 signalling and lymph node organogenesis in TRAF6-deficient mice. Genes Cells. 4:353-362.

13. Lomaga, M.A., et al. 1999. TRAF6 deficiency results in osteopetrosis and defective interleukin-1, CD40, and LPS signaling. Genes Dev. 13:1015-1024.

14. Iotsova, V., et al. 1997. Osteopetrosis in mice lacking NF-kappaB1 and NF-kappaB2. Nat. Med. 3:1285-1289.

15. Franzoso, G., et al. 1997. Requirement for NF-kap$\mathrm{paB}$ in osteoclast and B-cell development. Genes Dev. 11:3482-3496.

16. Xing, L., et al. 2002. NF-kappaB p50 and p52 expression is not required for RANK-expressing osteoclast progenitor formation but is essential for RANK- and cytokine-mediated osteoclastogenesis. J. Bone Miner. Res. 17:1200-1210.

17. Takayanagi, H., et al. 2002. Induction and activation of the transcription factor NFATc1 (NFAT2) integrate RANKL signaling in terminal differentiation of osteoclasts. Dev. Cell. 3:889-901.

18. Boyce, B.F., Yoneda, T., Lowe, C. Soriano, P., and Mundy, G.R. 1992. Requirement of pp60c-src expression for osteoclasts to form ruffled borders and resorb bone in mice. J. Clin. Invest. 90:1622-1627.

19. Grigoriadis, A.E., et al. 1994. c-Fos: a key regulator of osteoclast-macrophage lineage determination and bone remodeling. Science. 266:443-448.

20. Tondravi, M.M., et al. 1997. Osteopetrosis in mice lacking haematopoietic transcription factor PU.1. Nature. 386:81-84.

21. Pfeilschifter, J., Chenu, C., Bird, A., Mundy, G.R., and Roodman, G.D. 1989. Interleukin-1 and tumor necrosis factor stimulate the formation of human osteoclast-like cells in vitro. J. Bone Miner. Res. 4:113-118.

22. Goldring, S.R. 2003. Pathogenesis of bone and cartilage destruction in rheumatoid arthritis. Rheumatology. 42(Suppl. 2):ii1-ii6.

23. Kobayashi, K., et al. 2000. Tumor necrosis factor alpha stimulates osteoclast differentiation by a mechanism independent of the ODF/RANKLRANK interaction. J. Exp. Med. 191:275-286.

24. Lam, J., et al. 2000. TNF- $\alpha$ induces osteoclastogenesis by direct stimulation of macrophages exposed to permissive levels of RANK ligand. J. Clin. Invest. 106:1481-1488.

25. Boyce, B.F., Aufdemorte, T.B., Garrett, I.R., Yates, A.J., and Mundy, G.R. 1989. Effects of interleukin-1 on bone turnover in normal mice. Endocrinology. 125:1142-1150.

26. Uy, H.L., et al. 1995. Use of an in vivo model to determine the effects of interleukin-1 on cells at different stages in the osteoclast lineage. J. Bone Miner. Res. 10:295-301.

27. Fox, S.W., Fuller, K., and Chambers, T.J. 2000. Activation of osteoclasts by interleukin-1: divergent responsiveness in osteoclasts formed in vivo and in vitro. J. Cell Physiol. 184:334-340.

28. Erickson, S.L., et al. 1994. Decreased sensitivity to tumour-necrosis factor but normal T-cell development in TNF receptor 2-deficient mice. Nature. 372:560-563.

29. Peschon, J.J., et al. 1998. TNF receptor-deficient mice reveal divergent roles for p55 and p75 in several models of inflammation. J. Immunol. 160:943-952.

30. Abu-Amer, Y., et al. 2000. Tumor necrosis factor receptors types 1 and 2 differentially regulate osteoclastogenesis. J. Biol. Chem. 275:27307-27310.

31. Glaccum, M.B., et al. 1997. Phenotypic and functional characterization of mice that lack the type I 
receptor for IL-1. J. Immunol. 159:3364-3371.

32. Lorenzo, J.A., et al. 1998. Mice lacking the type I interleukin-1 receptor do not lose bone mass after ovariectomy. Endocrinology. 139:3022-3025.

33. Ducy, P., Zhang, R., Geoffroy, V., Ridall, A.L., and Karsenty, G. 1995. Osf2/Cbfal: a transcriptional activator of osteoblast differentiation. Cell. 89:747-754.

34. Ducy, P., and Karsenty, G. 1998. Genetic control of cell differentiation in the skeleton. Curr. Opin. Cell Biol. 10:614-619.

35. Marie, P.J. 2003. Fibroblast growth factor signaling controlling osteoblast differentiation. Gene. 316:23-32.

36. Roelen, B.A., and Dijke, P. 2003. Controlling mesenchymal stem cell differentiation by TGFBeta family members. J. Orthop. Sci. 8:740-748.

37. Blair, H.C., Zaidi, M., and Schlesinger, P.H. 2002. Mechanisms balancing skeletal matrix synthesis and degradation. Biochem. J. 364:329-341.

38. Olney, R.C. 2003. Regulation of bone mass by growth hormone. Med. Pediatr. Oncol. 41:228-234.

39. Kanis, J.A. 1998. Pathophysiology and treatment of Paget's disease of bone. 2nd edition. Martin Dunitz. London, United Kingdom. 310 pp.

40. Maldague, B., and Malghem, J. 1987. Dynamic radiologic patterns of Paget's disease of bone. Clin. Orthop. 217:126-251.

41. Huvos, A.G. 1986. Osteogenic sarcoma of bones and soft tissues in older persons. A clinicopathologic analysis of 117 patients older than 60 years. Cancer. 57:1442-1449.

42. Hansen, M.F., Nellissery, M.J., and Bhatia, P. 1999. Common mechanisms of osteosarcoma and Paget's disease. J. Bone Miner. Res. 14(Suppl. 2):39-44.

43. Hosking, D.J. 1981. Paget's disease of bone. Br. Med.J. (Clin. Res. Ed.). 283:686-688.

44. Alvarez, L., Peris, P., and Pons, F. 1997. Relationship between biochemical markers of bone turnover and bone scintigraphic indices in assessment of Paget's disease activity. Arthritis Rheum. 40:461-468.

45. Rebel, A., et al. 1981. Towards a viral etiology for Paget's disease of bone. Metab. Bone Dis. Relat. Res. 3:235-238.

46. Mills, B.G., Yabe, H., and Singer, F.R. 1988. Osteoclasts in human osteopetrosis contain viralnucleocapsid-like nuclear inclusions. J. Bone Miner. Res. 3:101-106.

47. Bianco, P., Silvestrini, G., Ballanti, P., and Bonucci, E. 1992. Paramyxovirus-like nuclear inclusions identical to those of Paget's disease of bone detected in giant cells of primary oxalosis. Virchows Arch. A, Pathol. Anat. Histopathol. 421:427-433.

48. Mills, B.G. 1981. Comparison of the ultrastructure of a malignant tumor of the mandible containing giant cells with Paget's Disease of bone. J. Oral Pathol. 10:203-215.

49. Menaa, C., et al. 2000. 1,25-dihydroxyvitamin D3 sensitivity of osteoclast precursors from patients with Paget's disease. J. Bone Miner. Res. 15:228-236.

50. Neale, S.D., Smith, R., Wass, J.A., and Athanasou, N.A. 2000. Osteoclast differentiation from circulating mononuclear precursors in Paget's disease is hypersensitive to 1,25-dihydroxyvitamin $\mathrm{D}(3)$ and RANKL. Bone. 27:409-416.

51. Menaa, C., et al. 2000. Enhanced RANK ligand expression and responsivity of bone marrow cells in Paget's disease of bone. J. Clin. Invest. 105:1833-1838.

52. Kurihara, N., et al. 2004. Role of TAFII-17, a VDR binding protein, in the increased osteoclast formation in Paget's disease. J. Bone Miner. Res. 19:1154-1164.

53. Sexton, P.M., Findlay, D.M., and Martin, T.J. 1999. Calcitonin. Curr. Med. Chem. 6:1067-1093.

54. Rogers, M.J. 2003. New insights into the molecular mechanisms of action of bisphosphonates. Curr. Pharm. Des. 9:2643-2658.

55. Miron-Canelo, J.A., Del Pino-Montes, J., Vicente-
Arroyo, M., and Saenz-Gonzalez, M.C. 1997. Epidemiological study of Paget's Disease of bone in a zone of the province of Salamanca (Spain). The Paget's Disease of the Bone Study Group of Salamanca. Eur. J. Epidemiol. 13:801-805.

56. Morales-Piga, A.A., Rey-Rey, J.S., Corres-Gonzalez, J., Garcia-Sagredo, J.M., and Lopez-Abente, G. 1995. Frequency and characteristics of familial aggregation of Paget's disease of bone. J. Bone Miner. Res. 10:663-670.

57. Montagu, M.F.A. 1949. Paget's disease (osteitis deformans) and hereditary. Am. J. Hum. Genet. 1:94-95.

58. Sofaer, J.A., Holloway, S.M., and Emery, A.E. 1983. A family study of Paget's disease of bone. J. Epidemiol. Community Health. 37:226-231.

59. Leach, R.J., Singer, F.R., Cody, J.D., and Roodman, G.D. 1999. Variable disease severity associated with a Paget's disease predisposition gene. J. Bone Miner. Res. 14:17-20.

60. Barker, D.J. 1981. The epidemiology of Paget's disease. Metab. Bone Dis. Relat. Res. 3:231-233.

61. Leach, R.J., Singer, F.R., and Roodman, G.D. 2001. The genetics of Paget's disease of the bone. J. Clin. Endocrinol. Metab. 86:24-28.

62. Cody, J.D., et al. 1997. Genetic linkage of Paget disease of the bone to chromosome 18q. Am. J. Hum. Genet. 61:1117-1122.

63. Haslam, S.I., et al. 1998. Paget's disease of bone: evidence for a susceptibility locus on chromosome $18 \mathrm{q}$ and for genetic heterogeneity. J. Bone Miner. Res. 13:911-917.

64. Laurin, N., et al. 2001. Paget disease of bone: mapping of two loci at 5q35-qter and 5q31. Am.J. Hum. Genet. 69:528-543.

65. Hocking, L.J., et al. 2001. Genomewide search in familial Paget disease of bone shows evidence of genetic heterogeneity with candidate loci on chromosomes 2q36,10p13, and 5q35. Am. J. Hum. Genet. 69:1055-1061.

66. Good, D.A., et al. 2002. Linkage of Paget disease of bone to a novel region on human chromosome 18q23. Am. J. Hum. Genet. 70:517-525.

67. Hughes, A.E., et al. 2000. Mutations in TNFRSF11A, affecting the signal peptide of RANK, cause familial expansile osteolysis. Nat. Genet. 24:45-48.

68. Nakatsuka, K., Nishizawa, Y., and Ralston, S.H. 2003. Phenotypic characterization of early onset Paget's disease of bone caused by a 27-bp duplication in the TNFRSF11A gene. J. Bone Miner. Res. 18:1381-1385.

69. Laurin, N., Brown, J.P., Morissette, J., and Raymond, V. 2002. Recurrent mutation of the gene encoding sequestosome 1 (SQSTM1/p62) in Paget disease of bone. Am. J. Hum. Genet. 70:1582-1588.

70. Johnson-Pais, T.L., et al. 2003. Three novel mutations in SQSTM1 identified in familial Paget's disease of bone. J. Bone Miner. Res. 18:1748-1753.

71. Hocking, L.J., et al. 2002. Domain-specific mutations in sequestosome 1 (SQSTM1) cause familial and sporadic Paget's disease. Hum. Mol. Genet. 11:2735-2739.

72. Hocking, L.J., et al. 2004. Novel UBA domain mutations of SQSTM1 in Paget's disease of bone: genotype phenotype correlation, functional analysis, and structural consequences. J. Bone Miner. Res. 19:1122-1127.

73. Park, I., et al. 1995. Phosphotyrosine-independent binding of a $62-\mathrm{kDa}$ protein to the src homology 2 (SH2) domain of p56lck and its regulation by phosphorylation of Ser-59 in the lck unique N-terminal region. Proc. Natl. Acad. Sci. U. S. A. 92:12338-12342.

74. Vadlamudi, R.K., Joung, I., Strominger, J.L., and Shin, J. 1996. p62, a phosphotyrosine-independent ligand of the $\mathrm{SH} 2$ domain of p56lck, belongs to a new class of ubiquitin-binding proteins. J. Biol. Chem. 271:20235-20237.
75. Shin, J. 1998. P62 and the sequestosome, a novel mechanism for protein metabolism. Arch. Pharm. Res. 21:629-633.

76. Ciani, B., Layfield, R., Cavey, J.R., Sheppard, P.W., and Searle, M.S. 2003. Structure of the ubiquitinassociated domain of p62 (SQSTM1) and implications for mutations that cause Paget's disease of bone. J. Biol. Chem. 278:37409-37412.

77. Geetha, T., and Wooten, M.W. 2002. Structure and functional properties of the ubiquitin binding protein p62. FEBS Lett. 512:19-24.

78. Duran, A., et al. 2004. The atypical PKC-interacting protein $\mathrm{p} 62$ is an important mediator of RANKactivated osteoclastogenesis. Dev. Cell. 6:303-309.

79. Kurihara, N., et al. 2004. The p392L mutation in the sequestasome-1 gene (p62) that is linked to Paget's disease (PD) is not sufficient to induce a pagetic phenotype in osteoclast (OCL) precursors [abstract]. J. Bone Miner. Res. 19:553.

80. Meunier, P.J., Coindre, J.M., Edouard, C.M., and Arlot, M.E. 1980. Bone histomorphometry in Paget's disease. Quantitative and dynamic analysis of pagetic and nonpagetic bone tissue. Arthritis Rheum. 23:1095-1103.

81. Laurin, N., Morissette, J., Raymond, V., and Brown, J.P. 2002. Large phenotypic variability of Paget disease of bone caused by the P392L sequestasome 1/p62 mutation [abstract]. J. Bone Miner. Res. 17:S380.

82. Whyte, M.P., et al. 2002. Osteoprotegerin deficiency and juvenile Paget's disease. N. Engl. J. Med. 18:175-184.

83. van Staa, T.P., et al. 2002. Incidence and natural history of Paget's disease of bone in England and Wales. J. Bone Miner. Res. 17:465-471.

84. Doyle, T. Gunn, J., Anderson, G., Gill, M., and Gundy, T. 2002 Paget's disease in New Zealand: evidence for declining prevalence. Bone. 31:616-619.

85. Abe, S., et al. 1995. Viral behavior of paracrystalline inclusions in osteoclasts of Paget's disease of bone. Ultrastruct. Pathol. 19:455-461.

86. Mills, B.G., et al. 1984. Evidence for both respiratory syncytial virus and measles virus antigens in the osteoclasts of patients with Paget's disease of bone. Clin. Orthop. 183:303-311.

87. Basle, M.F., Fournier, J.G., Rozenblatt, S., Rebel, A., and Bouteille, M. 1986. Measles virus RNA detected in Paget's disease bone tissue by in situ hybridization. J. Gen. Virol. 67:907-913.

88. Basle, M.F., et al. 1985. Paramyxovirus antigens in osteoclasts from Paget's bone tissue detected by monoclonal antibodies. J. Gen. Virol. 66:2103-2110.

89. Mills, B.G., et al. 1994. Multinucleated cells formed in vitro from Paget's bone marrow express viral antigens. Bone. 15:443-448.

90. Reddy, S.V., Singer, F.R., and Roodman, G.D. 1995. Bone marrow mononuclear cells from patients with Paget's disease contain measles virus nucleocapsid messenger ribonucleic acid that has mutations in a specific region of the sequence. J. Clin. Endocrinol. Metab. 80:2108-2111.

91. Friedrichs, W., et al. 2002. Sequence analysis of measles virus nucleocapsid transcripts in patients with Paget's disease. J. Bone Miner. Res. 17:145-157.

92. Birch, M.A., et al. 1994. Absence of paramyxovirus RNA in cultures of pagetic bone cells and in pagetic bone. J. Bone Miner. Res. 9:11-16.

93. Helfrich, M.H., et al. 2000. A negative search for a paramyxoviral etiology of Paget's disease of bone: molecular, immunological, and ultrastructural studies in UK patients. J. Bone Miner. Res. 15:2315-2329.

94. Kurihara, N., Reddy, S.V., Menaa, C., Anderson, D., and Roodman, G.D. 2000. Osteoclasts expressing the measles virus nucleocapsid gene display a pagetic phenotype. J. Clin. Invest. 105:607-614.

95. Reddy, S.V., et al. 2001. Osteoclasts formed by measles virus-infected osteoclast precursors from hCD46 transgenic mice express characteristics of 
pagetic osteoclasts. Endocrinology. 142:2898-2905. 96. Roodman, G.D., et al. 2004. The measles virus nucleocapsid (MVNP) gene is sufficient to induce a pagetic bone phenotype in vivo [abstract]. J. Bone Miner. Res. 19:54

97. Reddy, S.V., et al. 1999. Measles virus nucleocapsid transcript expression is not restricted to the osteoclast lineage in patients with Paget's disease of bone. Exp. Hematol. 27:1528-1532.

98. Gascon, G.G. 1996. Subacute sclerosing panencephalitis. Semin. Pediatr. Neurol. 3:260-269.

99. Marschall, M., Schuler, A., and Meier-Ewert, H 1996. Influenza C virus RNA is uniquely stabilized in a steady state during primary and secondary persistent infections. J. Gen. Virol. 77:681-686.

100.Lin, F., Mackay, D.K., and Knowles, N.J. 1998. The persistence of swine vesicular disease virus infection in pigs. Epidemiol. Infect. 121:459-472.

101.Anderson, D.C., and O’Driscoll, J.B. 1986. Dogs and Paget's disease [letter]. Lancet. 1:41.
102.Gordon, M.T., Anderson, D.C., and Sharpe, P.T 1991. Canine distemper virus localized in bone cells of patients with Paget's disease. Bone. 12:195-201.

103. Mee, A.P., et al. 1998. Detection of canine distemper virus in $100 \%$ of Paget's disease samples by in situ-reverse transcriptase-polymerase chain reaction. Bone. 23:171-175.

104. Roodman, G.D., et al. 1992. Interleukin 6. A potential autocrine/paracrine factor in Paget's disease of bone. J. Clin. Invest. 89:46-52.

105. Hoyland, J.A., Freemont, A.J., and Sharpe, P.T. 1994. Interleukin-6, IL-6 receptor, and IL-6 nuclear factor gene expression in Paget's disease. J. Bone Miner. Res. 9:75-80.

106.Neale, S.D., Schulze, E., Smith, R., and Athanasou, N.A. 2002. The influence of serum cytokines and growth factors on osteoclast formation in Paget's disease. QJM. 95:233-240.

107. Kurihara, N., Bertolini, D., Suda, T., Akiyama, Y., and Roodman, G.D. 1990. IL-6 stimulates osteoclast-like multinucleated cell formation in long-term human marrow cultures by inducing IL-1 release. J. Immunol. 144:4226-4230.

108.Tsurukai, T., Udagawa, N., Matsuzaki, K., Takahashi, N., and Suda, T. 2000. Roles of macrophagecolony stimulating factor and osteoclast differentiation factor in osteoclastogenesis. J. Bone Miner. Metab. 18:177-184.

109.Van Wesenbeeck, L., et al. 2002. The osteopetrotic mutation toothless (tl) is a loss-of-function frameshift mutation in the rat Csf1 gene: evidence of a crucial role for CSF-1 in osteoclastogenesis and endochondral ossification. Proc. Natl. Acad. Sci. U. S. A. 99:14303-14308.

110. Rubin, J., Fan, X., Thornton, D., Bryant, R, and Biskobing, D. 1996. Regulation of murine osteoblast macrophage colony-stimulation factor production by 1,25(OH)2D3. Calcif. Tissue Int. 59:291-296.

111. Troen, B.R. 2004. The role of cathepsin $\mathrm{K}$ in normal bone resorption. Drug News Perspect. 17:19-28. 